\title{
Signal processing in scanning thermoacoustic tomography in biological tissues
}

\author{
Yuan Xu and Lihong V. Wang ${ }^{\text {a) }}$ \\ Optical Imaging Laboratory, Biomedical Engineering Program, Texas A\&M University, 3120 TAMU, \\ College Station, Texas 77843-3120
}

(Received 12 December 2000; accepted for publication 27 March 2001)

\begin{abstract}
Microwave-induced thermoacoustic tomography was explored to image biological tissues. Short microwave pulses irradiated tissues to generate acoustic waves by thermoelastic expansion. The microwave-induced thermoacoustic waves were detected with a focused ultrasonic transducer to obtain two-dimensional tomographic images of biological tissues. The dependence of the axial and the lateral resolutions on the spectra of the signals was studied. A reshaping filter was applied to the temporal piezoelectric signals from the transducer to increase the weight of the high-frequency components, which improved the lateral resolution, and to broaden the spectrum of the signal, which enhanced the axial resolution. A numerical simulation validated our signal-processing approach. (C) 2001 American Association of Physicists in Medicine. [DOI: 10.1118/1.1380436]
\end{abstract}

Key words: microwave, ultrasonics, thermoacoustics, tomography, resolution, filter

\section{INTRODUCTION}

When electromagnetic radiation is absorbed in biological tissues, the heating and the subsequent expansion will cause emission of acoustic signals, which is called the thermoacoustic effect. In thermoacoustic tomography, the thermoacoustic signals from a tissue sample are collected to map the distribution of the radiative absorption within the sample. The radiative absorption is closely related to the physiological and pathological status of the tissue: for example, cancerous breast tissues are 2-5 times more strongly absorbing to microwaves than surrounding normal breast tissues, which has been attributed to an increase in bound water and sodium within malignant cells. ${ }^{1-3}$

Thermoacoustic tomography combines good imaging resolution with good imaging contrast. Purely microwave imaging has the advantage of good imaging contrast but suffers from poor spatial resolution due to the large wavelength of microwaves. ${ }^{4-7}$ On the other hand, purely ultrasonic imaging has good spatial resolution but poor contrast. Thermoacoustic tomography can bridge the gap between them.

There are various types of thermoacoustic tomography, such as photoacoustic tomography and microwave-induced thermoacoustic tomography (MITT). In photoacoustic tomography, ${ }^{8-11}$ due to the use of short laser pulses-several nanoseconds in pulse width - and the strong attenuation of the laser light by tissues, the frequency spectrum of the acoustic signal from the buried object of several micrometers in size is estimated to have significant components up to 75 $\mathrm{MHz},{ }^{9}$ which makes its axial resolution as good as $10 \mu \mathrm{m}$. However, the maximum imaging depth in photoacoustic tomography is limited by the strong attenuation of the laser light and of the high-frequency acoustic waves. On the other hand, MITT can be used to image much deeper tissues due to the relatively low absorption of microwaves. The spectra of the acoustic signals in MITT are usually below $2 \mathrm{MHz}$, and the axial resolution is greater than $1 \mathrm{~mm}$. Several investigators employed microwave-induced thermoacoustic waves in the 1980s for imaging of biological tissues; these early works, however, did not produce any tomographic or depthresolved images. ${ }^{12-14}$ Recent progress realized tomographic imaging of biological tissues based on microwave-induced thermoacoustic waves. ${ }^{15-18}$

We here present our studies on the signal-processing aspect of scanning MITT. Filtering has been applied to signal processing in photoacoustic tomography ${ }^{11}$ and MITT; $;{ }^{15}$ however, it was used to eliminate the dc (direct current) offset and the effect of the response of the transducer on the piezoelectric signal, respectively. For the first time, we discuss in details how the spectra of signals influence the resolution of thermoacoustic tomography and how the resolution can be improved by signal processing. In our imaging approach, the lateral resolution was achieved by use of a focused ultrasonic transducer, whereas the axial resolution was obtained by measuring the temporal profiles of the acoustic signals. The dominance of the low-frequency $(<0.5 \mathrm{MHz})$ components in the raw temporal signals limited the lateral resolution, and the narrow bandwidth of the signals restricted the axial resolution. Consequently, the image before signal processing had poor lateral resolution and many artifacts. We showed that a "simple" filtering method improved the lateral resolution to some extent but at the expense of the axial resolution. To overcome this problem, we proposed a new reshaping filter. It was applied to the temporal signals from the transducer to increase the weight of the high-frequency components, which improved the lateral resolution, and to broaden the spectrum of the signal, which enhanced the axial resolution. A numerical simulation validated our signal-processing approach. 


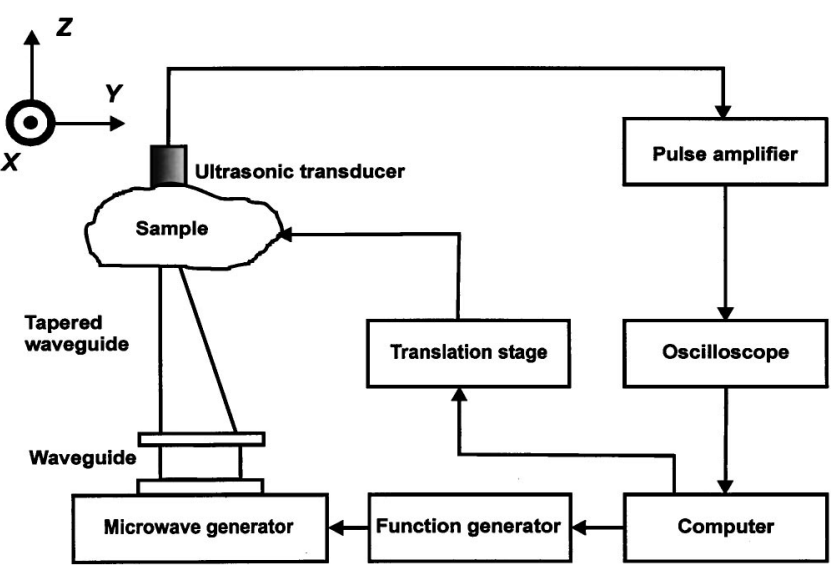

FIG. 1. Experimental setup for scanning MITT.

\section{METHODS}

\section{A. Experimental setup}

The experimental setup for this study is shown in Fig. 1. A Cartesian coordinate system was set up for reference: The $x$ axis pointed outward perpendicularly to the drawing plane, the $y$ axis pointed to the right in the drawing plane, and the $z$ axis pointed upward along the acoustic axis. A $3 \mathrm{GHz}$ microwave generator transmitted microwave pulses. The pulse width was modified from the original manufacturer's setting to $0.5 \mu \mathrm{s}$. A function generator was employed to trigger the microwave generator, to control its pulse repetition frequency, and to synchronize the sampling by the oscilloscope. Microwave energy was delivered by a tapered waveguide with a cross section that gradually narrowed from $72 \mathrm{~mm}$ $\times 34 \mathrm{~mm}$ to $72 \mathrm{~mm} \times 5 \mathrm{~mm}$. The object to be imaged was a slab of chicken muscle with a $y-z$ cross section of $14 \mathrm{~mm}$ $\times 8 \mathrm{~mm}$, and the slab was plunged into lard contained in a plexiglass tank. The tank was mounted on a two-dimensional (2D) $x-y$ translation stage (MD2, Arrick Robotics), which was driven by two computer-controlled stepper motors. Lard and plexiglass were used for their low absorption to microwaves. Lard also provided good acoustic coupling to an ultrasonic transducer facing the microwave waveguide. The central frequency of the ultrasonic transducer (V314, Panametrics) was $1 \mathrm{MHz}$, the bandwidth was $0.6 \mathrm{MHz}$, the diameter of the active element was $1.9 \mathrm{~cm}$, and the focal length was $2.5 \mathrm{~cm}$. The transducer was connected to a low-noise pulse preamplifier. The amplified signal was averaged 100 times, recorded by an oscilloscope (TDS-640A, Tektronix), and then transferred to a personal computer.

In our scanning MITT, the ultrasonic transducer measured the time-of-arrival signals of the thermoacoustic waves. The distances between the thermoacoustic sources and the transducer were calculated by multiplying the time of arrival with the speed of sound in the medium. Therefore, a time-domain signal can be converted into a one-dimensional (1D) image along the acoustic axis ( $z$ axis), which is similar to an ultrasonic A-scan image. Scanning the sample along the $x$ or the $y$ axis and combining the multiple 1D images yielded a $2 \mathrm{D}$ cross-sectional image of the sample in the $x-z$ or $y-z$ plane, which is analogous to an ultrasonic B-scan image.

\section{B. Signal processing}

Two methods of signal processing in the frequency domain based on finite impulse response (FIR) filters were applied to the experimental data. The filtering is implemented by multiplying a properly selected real window function to the spectra of the signals, which introduces no phase distortion. In the first method, all the signals are processed with the same bandpass filter, which has a passband between 0.5 and $1.5 \mathrm{MHz}$ with a transition bandwidth of $\sim 0.5 \mathrm{MHz}$.

To overcome the difficulties of the first method, we proposed a reshaping method in the frequency domain. This method can make the bandwidth of the processed signal broader to enhance the axial resolution and weigh the highfrequency components more heavily to improve the lateral resolution. The essence of this method is to apply a reshaping filter to each temporal signal. The shape of the reshaping filter for the signal at any $y$ position is chosen to be the inverse of the envelope of the original frequency spectrum, where the envelope of the spectrum is obtained by connecting the major local maxima. Without distorting the positions of the pulses in the temporal signal, this filter can achieve the widest possible bandwidth in the filtered signal and consequently the best axial resolution. Moreover, to filter out the very low-frequency disturbance-which is caused by the preamplifier-and the high-frequency noise beyond the cutoff frequency-where the signal-to-noise ratio is unity, a smoothing filter is applied to the signal. Unlike the reshaping filter, the smoothing filter is the same for the signals from all the scanned positions of the transducer. The final filter is the product of the above two filters in the frequency domain. To increase the contrast, the background value is subtracted from the spectrum before the final filter is applied. Lastly, to improve the lateral resolution, the final filter is scaled by a constant factor such that the spectral amplitudes of all the piezoelectric signals at a selected high frequency remain unchanged after filtering.

\section{RESULTS AND DISCUSSION}

An image of the chicken muscle is presented in Fig. 2(a). Each vertical line in this $2 \mathrm{D}$ image was obtained from a temporal piezoelectric signal of the ultrasonic transducer, and the sample was scanned horizontally along the $y$ axis with a step size of $1 \mathrm{~mm}$ to acquire the multiple vertical lines. Figures 2(b) and 2(c) show the temporal wave forms and the corresponding frequency spectra, respectively, for $y$ equal to $20 \mathrm{~mm}$-where the transducer axis crossed the muscle - and equal to $2 \mathrm{~mm}$ - where the transducer axis did not cross the muscle. The buried muscle was clearly imaged as shown in Fig. 2(a): The white line at $z=17 \mathrm{~mm}$ corresponds to the upper boundary between the lard and the muscle, and the dark line at $z=25.1 \mathrm{~mm}$ to the lower one. The thickness of the muscle in the image is $8.1 \mathrm{~mm}$ and agrees with the actual one. But the lateral resolution is poor: The width of the muscle in the image appears to be $36 \mathrm{~mm}$, 
(a)

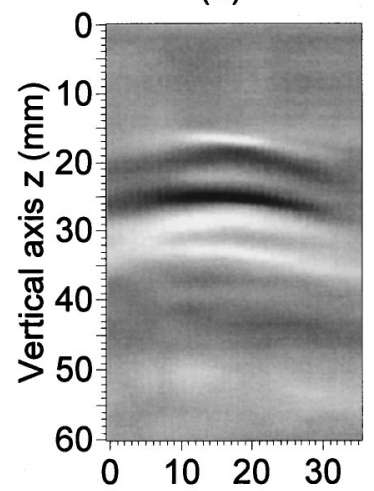

Horizontal axis y $(\mathrm{mm})$

\section{$\begin{array}{lll}-0.100 & -0.025 & 0.050\end{array}$}

Piezoelectric signal (a.u.) (b)

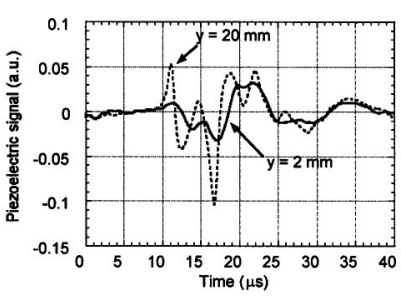

(c)

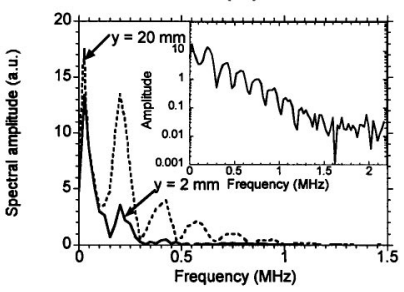

FIG. 2. (a) 2D image of the $y-z$ cross section of the sample obtained by scanning MITT before data processing; (b) temporal microwave-induced thermoacoustic signals at different positions $y=20$ and $2 \mathrm{~mm}$; (c) the main panel shows both of the corresponding spectra on a linear scale, and the inset shows the spectrum for $y=20 \mathrm{~mm}$ on a logarithmic scale.

much greater than the actual $14 \mathrm{~mm}$ width. Furthermore, there appear many ghost objects between the two boundaries and below the lower boundary. Because the muscle and the lard are almost uniform, no heterogeneity in the image is expected from other than the boundaries.

To explain these problems, we resort to the relationship between the lateral resolution of the detecting ultrasonic transducer and the frequency spectrum of the received temporal wave form. The lateral resolution of the ultrasonic transducer is determined by its focal diameter, which is given by

$$
d_{f}=\frac{1.02 \nu_{a} l_{f}}{d_{a} f_{a}},
$$

where $\nu_{a}$ is the speed of sound in the medium, $l_{f}$ is the focal length of the transducer, $d_{a}$ is the diameter of the active element in the transducer, and $f_{a}$ is the acoustic frequency.

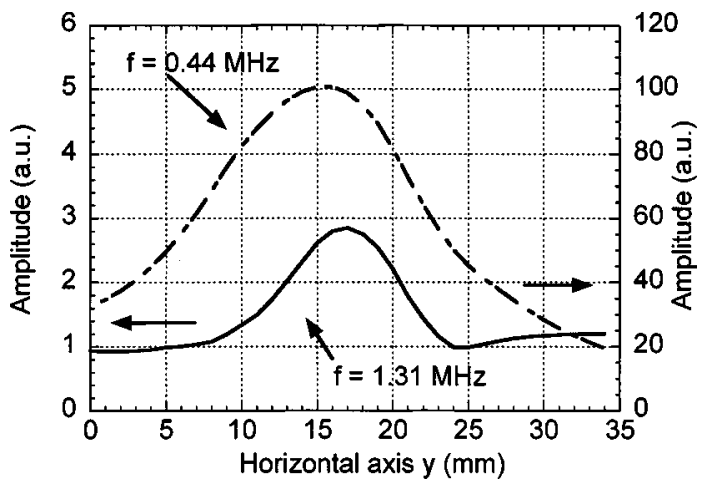

FIG. 3. The distributions of the signal components around $f=1.31$ and 0.44 MHz along the $y$ axis.

(a)
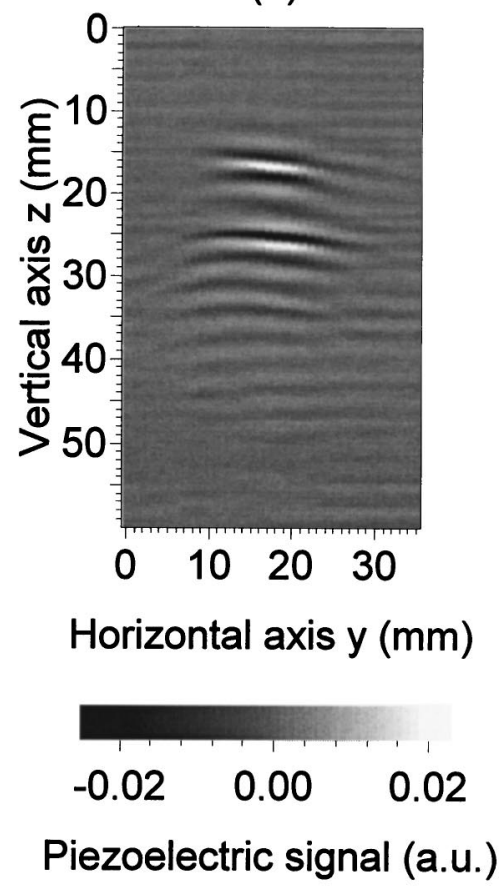

(b)

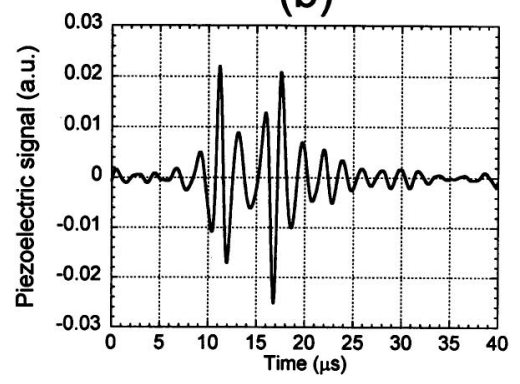

FIG. 4. (a) The 2D image after filtering the temporal signals with a standard bandpass filter; (b) the filtered temporal signal at $y=20 \mathrm{~mm}$.

Therefore, the lateral resolution is inversely proportional to the frequency of the acoustic signal or the piezoelectric signal. Because the dominant frequency components of the piezo-electrical signals are far below $1 \mathrm{MHz}$, as shown in Fig. 2(c), the lateral resolution is much worse than the focal diameter of the transducer, $2 \mathrm{~mm}$, at its $1 \mathrm{MHz}$ central frequency.

The dependence of the lateral resolution on the frequency spectra is illustrated more clearly in Fig. 3, which displays the 1D lateral images-along the $y$ axis-corresponding to the 1.31 and $0.44 \mathrm{MHz}$ components of the spectra, respectively. The $1.33 \mathrm{MHz}$ image is sharper than the $0.44 \mathrm{MHz}$ one, and therefore, has superior lateral resolution. Poor resolution that is caused by the dominating low-frequency components is also responsible for the ghost piezoelectric signals at $y=2 \mathrm{~mm}$, where the transducer axis does not cross the buried muscle and thus the received piezoelectric signals can be only wide-angle low-frequency signals. In comparison, the piezoelectric signal at $y=20 \mathrm{~mm}$ - where the acoustic axis of the transducer crosses the muscle-is primarily from the transducer axis and hence has greater high-frequency 
(a)

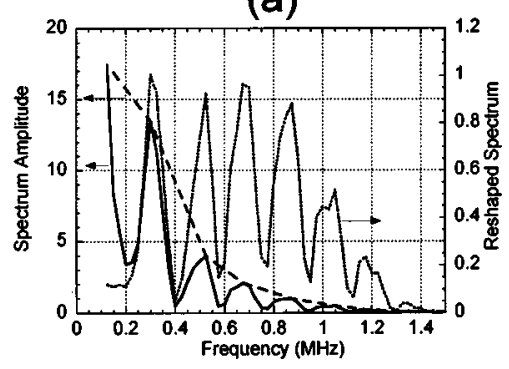

(c)

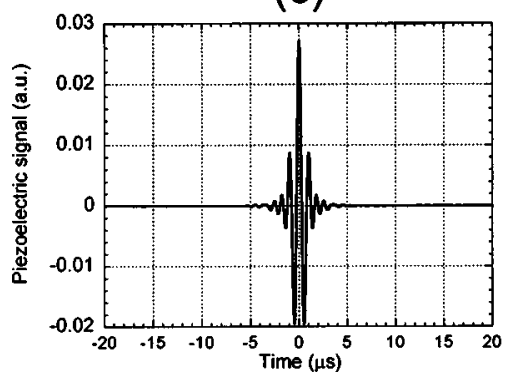

(b)

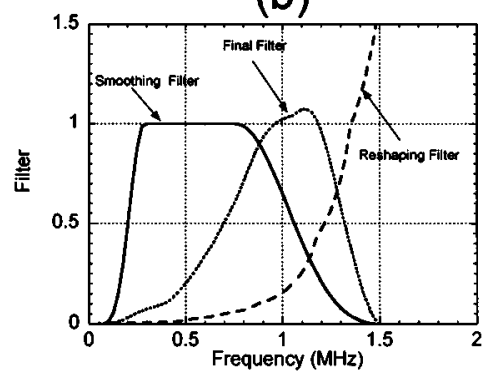

(d)

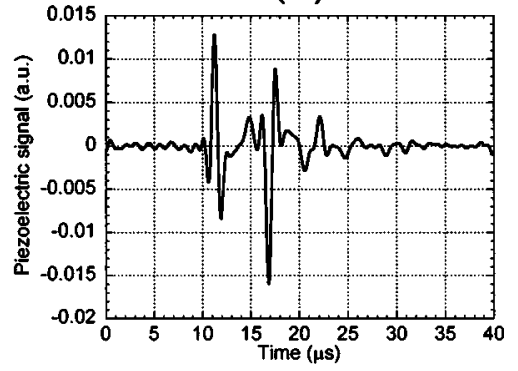

FIG. 5. (a) The envelope (dashed curve) of the spectrum (solid curve) of the temporal signal at $y=20 \mathrm{~mm}$ and the spectrum after the reshaping processing (dotted curve); (b) the reshaping filter-which is the inverse of the envelope, the smoothing filter-which is used to filter the high-frequency noise and the extremely lowfrequency signal, and the final filter-which is the product of the reshaping filter and the smoothing filter; (c) the temporal wave form corresponding to the final filter; (d) the temporal wave form corresponding to the spectrum in Fig. 5(a) after the reshaping processing. components than the piezoelectric signal at $y=2 \mathrm{~mm}$, as shown in Fig. 2(c).

From the above discussion, it is clear that increasing the high-frequency components of the piezoelectric signals can improve the lateral resolution. The most natural solution is to apply a bandpass filter to cut off the low-frequency components. An example of such processing, in which the filter has a passband between 0.5 and $1.5 \mathrm{MHz}$ with a transition bandwidth of $\sim 0.5 \mathrm{MHz}$, is shown in Fig. 4(a). The lateral resolution is much improved but still unsatisfactory; however, the axial resolution seems worse, and some artifacts were generated, as shown in Fig. 4(b). The poor axial resolution is due to the decrease of bandwidth in the signal processing. As shown in the inset of Fig. 2(c), the spectral amplitude drops exponentially with the frequency; therefore, the filtered signal has a narrower bandwidth than the original one. A narrower bandwidth in the frequency domain results in a broader signal in the time domain thus poor axial resolution.

To overcome the difficulties of the simply filtering, we applied reshaping filters to the signals. The shape of the reshaping filter [Fig. 5(b)] for the signal at $y=20 \mathrm{~mm}$ is chosen to be the inverse of the envelope of the original frequency spectrum; where the envelope of the spectrum is obtained by connecting the major local maxima, as shown by the dashed curve in Fig. 5(a). Without distorting the positions of the pulses in the temporal signal, this filter can achieve the widest possible bandwidth in the filtered signal and consequently the best axial resolution. Moreover, to filter out the very low-frequency disturbance-which is caused by the preamplifier-and the high-frequency noise beyond the cutoff frequency-where the signal-to-noise ratio is unity, a smoothing filter is applied to the signal. Unlike the reshaping filter, the smoothing filter is the same for the signals from all the scanned positions of the transducer.

The final filter, which is the product of the above two filters in the frequency domain, is shown in Fig. 5(b); the final filter in the time domain is shown in Fig. 5(c). To increase the contrast, the background value-which is determined by the amplitude at both the ends of the solid curve in Fig. 3-is subtracted from the spectrum before applying the final filter. As we wish to obtain a lateral resolution approaching that at $1.31 \mathrm{MHz}$ (Fig. 3), the final filter is scaled by a constant factor such that the spectral amplitude of the final filter at $1.31 \mathrm{MHz}$ is set to unity; consequently, the spectral amplitudes of the piezoelectric signals for all piezoelectric signals remain unchanged at $1.31 \mathrm{MHz}$. The spectrum and the temporal wave form of the processed signal at $y=20 \mathrm{~mm}$ are displayed in Figs. 5(a) and 5(d). The axial resolution of the processed signal is much better than that of the unprocessed signal [Fig. 2(b)] because the processed spectrum is much broader than the unprocessed one [Fig. 2(c)]. The processed 2D image (Fig. 6) is also clearer than the original image. The ghost objects in the original images were removed, and the two boundaries became quite distinct from the background. The muscle along the $y$ axis in the image is about $15 \mathrm{~mm}$, which agrees well with the $14 \mathrm{~mm}$ actual size.

A numerical simulation was implemented to test our reshaping method. A simulated temporal waveform [Fig. 7(a)] includes three pulses at $t=20,21$, and $30 \mu$ s. The 20 and 30 $\mu$ s pulses are determined by

$$
s(t)=\exp \left(-\frac{\left(t-t_{0}\right)^{2}}{w^{2}}\right),
$$

and the $21 \mu$ s pulse is determined by

$$
s(t)=1 /\left(1+\frac{\left(t-t_{0}\right)^{2}}{w^{2}}\right),
$$

where $w$ is the pulse width and is set to $0.8 \mu$ s. The corresponding spectrum is shown in Fig. 7(b), which resembles the spectrum of the signal at $y=20 \mathrm{~mm}$ in our image. Be- 


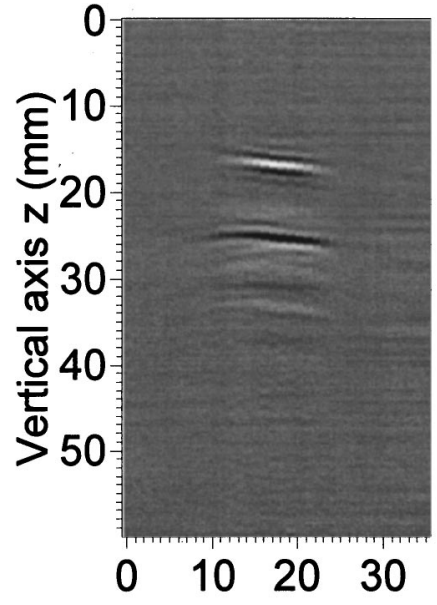

Horizonal axis y $(\mathrm{mm})$

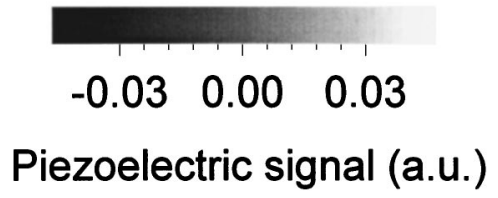

FIG. 6. The 2D image after applying the final filter to the temporal piezoelectric signals.

cause the 20 and $21 \mu$ s pulses are too close to each other, they merge into one pulse in the original constructed signal. In the signal processed by our method [Fig. 7(c)], the two pulses are separated distinctively, and all the pulses become sharper. The positioning errors of the restored peaks are within $10 \%$ of the pulse width. However, the signal-to-noise ratio (SNR) in Fig. 7(c) is lower than that in Fig. 7(a) as a result of the increased weighting of the noisy high-frequency components in the reshaping process. Nevertheless, the degradation of SNR in the processed signals has little influence on the image, as shown in Fig. 6. In contrast, the simple filtering did not produce as a good outcome [Fig. 7(d)].

Our reshaping method is especially efficient for signals that consist of multiple pulses of similar shapes, which is quite common in ultrasonic detection. Assuming a temporal signal comprises two identical pulses-for illustration purposes - at different times, its spectral amplitude can be written as

$$
A(f)=s(f)|1+\exp (i 2 \pi f \Delta t)|=s(f) p(f),
$$

where $f$ is the frequency, $s(f)$ is the spectral amplitude of a single pulse, and $\Delta t$ is the time interval between the two pulses. Because the oscillatory $p(f)$ has a flat envelope, the envelope of $A(f)$ approximately equals that of $s(f)$, which determines the shape of a single pulse. On the other hand, the features and the phase of the spectrum contain the positioning information of the pulses, which is the most important information in imaging. In our reshaping method, the signal is multiplied by the reciprocal of its own spectral envelope, resulting in a flat envelope in the processed spectrum; consequently, the pulses are narrowed in the time domain. As the reshaping filter is smooth, it does not alter the features and the phase of the spectrum; accordingly, the positions of the pulses in the time domain remain unchanged.

In general, applying the reshaping filter will sharpen the boundaries of signals, which can be illustrated with an ideal slab. The thermoacoustic wave from a slab irradiated by a sufficiently short microwave pulse can be represented by a square wave. It can be shown that filtering the square wave with the reshaping filter is equivalent to taking the first derivative of the wave in the time domain, which yields the two boundaries of the slab. The spectral amplitude of the square wave with a duration $a$ is $|\sin (\pi a f) /(\pi f)|$; thus its envelope is $1 /(\pi f)$, and the reshaping filter is $\pi f$. Applying the reshaping filter to the square wave in the frequency domain is (a)

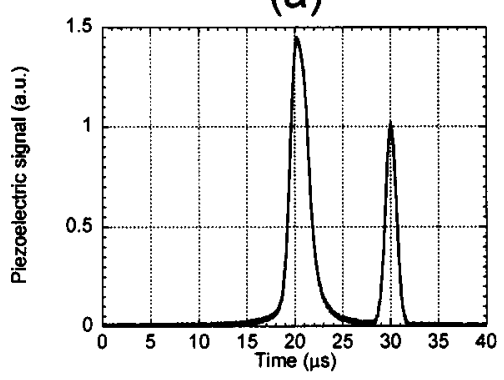

(c)

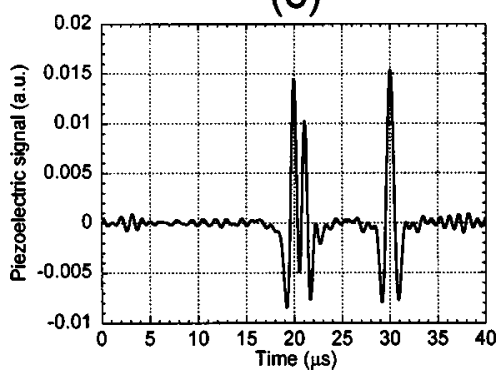

(b)

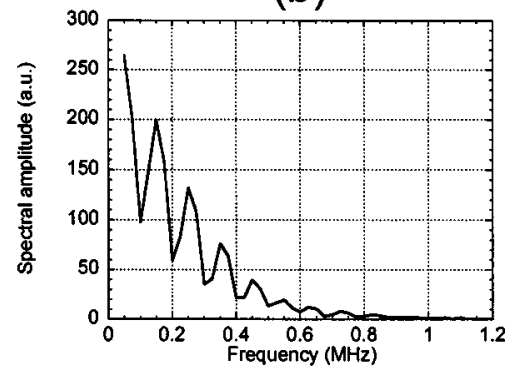

(d)

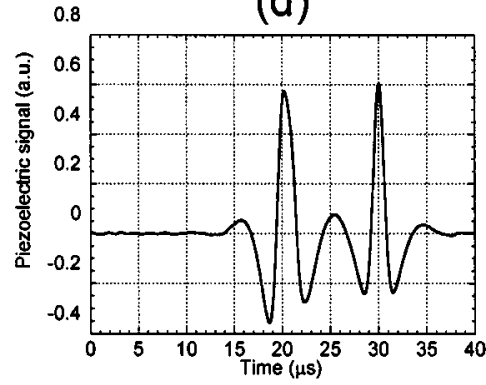

FIG. 7. (a) The simulated piezoelectric signal; (b) the corresponding spectrum; (c) the piezoelectric signal after reshaping the spectrum; (d) the piezoelectric signal after applying the smoothing filter as shown in Fig. 5 (b). 
equivalent, within a constant factor, to differentiating the signal in the time domain, which yields a positive delta function at the front boundary of the square wave and a negative one at the rear boundary.

Our study pointed out some potential approaches that can be used to improve the imaging resolution in our experiments. One approach is to improve the SNR of the signal so that the cutoff frequency is extended. Because only the spectral region with an SNR greater than unity provides useful information for our reshaping method, an increased cutoff frequency can broaden the usable spectrum and accordingly improve both the lateral and the axial resolutions. However, this advantage can only be realized when the spectrum is reshaped because the unprocessed high-frequency spectral amplitude is so small compared with the low-frequency one that it contributes little to improving the lateral and the axial resolutions, as shown in Fig. 2(c); after the reshaping, the weight of the high-frequency portion is increased greatly, resulting in an improved resolution. Another potential approach to improving the resolution is to shift the acoustic spectrum to a higher frequency by modulating the microwave source.

\section{CONCLUSIONS}

Our studies showed that scanning MITT is a promising imaging tool for biological tissues. The boundaries of different tissue constituents can be imaged clearly and accurately with the assistance of image processing. By reshaping the spectra of the piezoelectric signals, the weight of the highfrequency components is increased greatly, resulting in much improved axial and lateral resolutions, both of which were 1 $\mathrm{mm}$ in our current experimental setup. The numerical simulation also verified our signal-processing method. Our spectral-reshaping method can also be applied to other ultrasonic signals comprising several pulses of similar shapes.

\section{ACKNOWLEDGMENTS}

We thank G. Ku for experimental assistance. This project was sponsored in part by the U.S. Army Medical Research and Materiel Command Grant No. DAMD17-00-1-0455, the National Institutes of Health Grants No. R01 CA71980 and No. R21 CA83760, the National Science Foundation Grant No. BES-9734491, and Texas Higher Education Coordinating Board Grant No. ARP 000512-0123-1999.
${ }^{\text {a) }}$ Author to whom all correspondence should be addressed. Telephone: 979847-9040; Fax: 979-845-4450; Electronic mail: LWang@tamu.edu; URL:http://oilab.tamu.edu

${ }^{1}$ W. Joines, R. Jirtle, M. Rafal, and D. Schaeffer, "Microwave power absorption differences between normal and malignant tissue," Int. J. Radiat. Oncol., Biol., Phys. 6, 681-687 (1980).

${ }^{2}$ S. Chaudhary, R. Mishra, A. Swarup, and J. Thomas, "Dielectric properties of normal human breast tissues at radiowave and microwave frequencies," Indian J. Biochem. Biophys. 21, 76-79 (1984).

${ }^{3}$ W. Joines, Y. Zhang, C. Li, and R. Jirtle, "The measured electrical properties of normal and malignant human tissues from $50-900 \mathrm{MHz}$," Med. Phys. 21, 547-550 (1994).

${ }^{4}$ Medical Applications of Microwave Imaging, edited by L. E. Larsen and J. H. Jacobi (IEEE, Piscataway, NJ, 1986).

${ }^{5}$ S. Caorsi, A. Frattoni, G. L. Gragnani, E. Nortino, and M. Pastorino, "Numerical algorithm for dielectric-permittivity microwave imaging of inhomogeneous biological bodies," Med. Biol. Eng. Comput. 29, NS37NS44 (1991).

${ }^{6}$ M. S. Hawley, A. Broquetas, L. Jofre, J. C. Bolomey, and G. Gaboriaud, "Microwave imaging of tissue blood content changes," J. Biomed. Eng. 13, 197-202 (1991).

${ }^{7}$ P. M. Meaney, K. D. Paulsen, and J. T. Chang, "Near-field microwave imaging of biologically-based materials using a monopole transceiver system," IEEE Trans. Microwave Theory Tech. 46, 31-45 (1998).

${ }^{8}$ R. A. Kruger, P. Liu, Y. R. Fang, and C. R. Appledorn, "Photoacoustic ultrasound (PAUS) - reconstruction tomography," Med. Phys. 22, 16051609 (1995).

${ }^{9}$ C. G. A. Hoelen, F. F. M. Demul, R. Pongers, and A. Dekker, "Threedimensional photoacoustic imaging of blood vessels in tissue," Opt. Lett. 23, 648-650 (1998).

${ }^{10}$ A. A. Karabutov, E. V. Savateeva, N. B. Podymova, and A. A. Oraevsky, "Backward mode detection of laser-induced wide-band ultrasonic transients with optoacoustic transducer," J. Appl. Phys. 87, 2003-2014 (2000).

${ }^{11}$ C. G. A. Hoelen, R. Pongers, G. Hamhuis, F. F. M. Demul, and J. Greve, "Photoacoustic blood cell detection and imaging of blood vessels in phantom tissue," Proc. SPIE 3196, 142-153 (1998).

${ }^{12}$ T. Bowen, L. Nasoni, A. E. Pifer, and G. H. Sembrosk, "Some experimental results on the thermoacoustic imaging of soft tissue-equivalent phantoms," Proc.-IEEE Ultrason. Symp. 2, 823-827 (1981).

${ }^{13}$ R. G. Olsen and J. C. Lin, "Acoustic imaging of a model of a human hand using pulsed microwave irradiation," Bioelectromagnetics (N.Y.) 4, 397-400 (1983).

${ }^{14}$ J. C. Lin and K. H. Chan, "Microwave thermoelastic tissue imagingsystem design," IEEE Trans. Microwave Theory Tech. 32, 854-860 (1984).

${ }^{15}$ R. A. Kruger, D. R. Reinecke, and G. A. Kruger, "Thermoacoustic computed tomography-technical considerations," Med. Phys. 26, 18321837 (1999).

${ }^{16}$ L.-H. V. Wang, X. Zhao, H. Sun, and G. Ku, "Microwave-induced acoustic imaging of biological tissues," Rev. Sci. Instrum. 70, 3744-3748 (1999).

${ }^{17} \mathrm{G}$. Ku and L.-H. V. Wang, "Scanning thermoacoustic tomography in biological tissue," Med. Phys. 27, 1195-1202 (2000).

${ }^{18}$ G. Ku and L.-H. V. Wang, "Scanning microwave-induced thermoacoustic tomography: signal, resolution, and contrast," Med. Phys. 28, 4-10 (2001). 